\title{
RELACIONES ENTRE EL DESARROLLO MORFOSINTÁCTICO Y LA TEORÍA DE LA MENTE: ESTUDIO DE CASO DE UN NIÑNO CON SÍNDROME DE ASPERGER
}

\author{
CECILIA LATORRE COSCULLUELA* \\ UNIVERSIDAD DE ZARAGOZA, ESPAÑA
}

Recibido: 27 de Octubre de 2015

Aprobado: 15 de Abril de 2016

Para referenciar este artículo:

Latorre Cosculluela, C. (2016). Relaciones entre el desarrollo morfosintáctico y la teoría de la mente: estudio de un caso de un niño con síndrome de asperger. Revista Iberoamericana de Psicología: Ciencia y Tecnología, 9 (1), 9- 17

\begin{abstract}
Resumen
La evidencia empírica recogida hasta la fecha ofrece argumentos a favor del importante papel que desempeña la dimensión morfosintáctica del lenguaje en el desarrollo de las habilidades de Teoría de la Mente. No obstante, un gran porcentaje de estas investigaciones se ha llevado a cabo con poblaciones de niños con desarrollo típico, por lo que se requieren más estudios que analicen estos aspectos en otras poblaciones, por ejemplo, en niños con Síndrome de Asperger. El objetivo de este trabajo ha sido examinar el desarrollo morfosintáctico y su relación con la adquisición de habilidades de Teoría de la Mente en un niño con Síndrome de Asperger, a través de un estudio de caso $(n=1)$ de corte cualitativo, con enfoque interpretativo. Los resultados obtenidos indican que el mayor número de errores morfosintácticos detectados en el participante se relacionan con la construcción de complementos oracionales y la utilización de pronombres personales. Se estudiaron, además, las habilidades narrativas, ya que estas constituyen un factor clave en el desempeño de tareas que implican directamente el desarrollo de capacidades de Teoría de la Mente. La evaluación de las secuencias narrativas registradas permitió notar que un gran porcentaje de errores morfosintácticos se presenta en tareas que implican el uso de habilidades de Teoría de la Mente, mientras que en aquellas historias que no comprometen la ejecución de dichas habilidades no solo se presentan menos errores sino que, además, las narraciones son más ricas y completas.

Palabras clave. Síndrome de Asperger, desarrollo morfosintáctico, Teoría de la Mente, habilidades narrativas.
\end{abstract}

\section{THE RELATIONSHIP BETWEEN MORPHO-SYNTACTIC DEVELOPMENT AND THEORY OF MIND: A CASE STUDY OF A CHILD WITH ASPERGER SYNDROME.}

\begin{abstract}
Empirical evidence gathered to date supports arguments in favor of the undoubtable role of the morpho-syntactical dimension of language on the development of skills of a Theory of Mind. However, a large percentage of the research has been carried out with populations of children with typical development, thus more studies are required, especially ones that analyze these aspects in other populations, for instance, children with Asperger Syndrome. Accordingly, the aim of this study was to examine morphological and syntactic development, and its relation to the acquisition of Theory of Mind skills in a child with Asperger Syndrome, through a qualitative case study $(n=1)$ with an interpretative approach. The results obtained indicate that the largest number of morpho-syntactic errors detected in the the child are related to the construction of sentential complements and to the use of personal pronouns. In addition, narrative skills were also studied, since they constitute an essential factor in the performance of tasks that directly imply the development of skills of Theory of Mind. Evaluation of the registered narrative sequences allowed to see that a large percentage of morphosyntactic errors show up in tasks that require the direct implication of Theory of Mind skills; meanwhile, less errors show up in stories that don't imply the execution of these skills, and their narratives are much richer and complete. Keywords. Asperger Syndrome, morphological and syntactic development, Theory of Mind, narrative skills.
\end{abstract}

* Doctoranda en Educación. Máster Universitario en Estudios avanzados sobre el Lenguaje, la Comunicación y sus Patologías. Universidad de Zaragoza. Facultad de ciencias humanas y de la educación. cecilialacos@gmail.com 
Los estudios experimentales sobre el desarrollo de las habilidades que permiten a los niños entender lo que ocurre en la propia mente y la de los otros, reflejan un consenso general que reconoce la importancia de la comprensión de estados mentales (emociones, intenciones, creencias, etc.) (Hughes y Dunn, 1997). A esta habilidad para conocer la mente de los demás se la ha denominado Teoría de la Mente (ToM, en adelante, por sus siglas en ingles) y ha constituido un aspecto muy analizado desde el campo de la psicología. Este constructo refiere, bajo la opinión de Tirapu-Ustárroz, Pérez-Sayes, Erekatxo-Bilbao \& Pelegrín-Valero (2007), a la habilidad para comprender y predecir la conducta de otras personas, sus conocimientos, sus intenciones y sus creencias. Los trabajos en esta área han situado el foco de atención en la comprensión que tienen los niños de sí mismos y de otras personas como seres mentales, es decir, como seres que tienen creencias, deseos e intenciones que, al ser interpretadas, pueden oficiar como causa de las acciones observadas (Milligan, Astington \& Dack 2007).

La numerosa evidencia empírica recogida hasta la fecha señala que, tanto en niños con desarrollo típico como en muestras clínicas (niños autistas, con Trastorno Específico del Lenguaje, con sordera, etc.), son las capacidades lingüísticas las más ampliamente correlacionadas con las habilidades de teoría de la mente, especialmente las implicadas en el desarrollo de tareas que implican la comprensión de falsas creencias o falsos conocimientos (Cutting \& Dunn, 1999 citado por Resches, Serrat, Rostan \& Esteban, 2010). El desempeño registrado en esta clase de tareas, además de constituir un elemento esencial que marca la aparición de la ToM en el desarrollo típico, se ha tomado como un indicador del déficit que parece tomar lugar en niños que han sido diagnosticados dentro del espectro autista (Baron-Cohen, Leslie \& Frith, 1985).

Así pues, de la misma manera que los niños aprenden acerca de la biología o la física, el contenido de lo que llamamos ToM se transporta a través del lenguaje (Hughes \& Dunn, 1997). Sin embargo, en los últimos años ha habido un intenso debate sobre la naturaleza de esta relación, en particular, acerca de si es posible afirmar una suerte de relación de causalidad entre la ToM y habilidades lingüísticas. (Fisher, Happé \& Dunn, 2005). En otras palabras, aunque entre los dos y los cuatro años de edad (momento en que se suele situar la aparición de la ToM en el desarrollo), son múltiples las conexiones entre el desarrollo conceptual y el lingüístico, no está del todo claro si el lenguaje engendra al pensamiento o si, por el contrario, las habilidades lingüísticas dependen de la aparición previa de un repertorio de habilidades mentalistas (ToM) especificas.
De acuerdo con Fisher, Happé \& Dunn (2005), existen tres posibilidades básicas explicativas de la relación entre el lenguaje y la ToM. En primer lugar, puede afirmarse que el lenguaje puede ser facilitador del desarrollo de una ToM; en segundo lugar, que una ToM puede ser necesaria para el desarrollo del lenguaje; $y$, finalmente, que no existe una relación causal directa en la relación observada. En esta misma línea, Bermúdez (2009), en un artículo de reflexión y revisión de antecedentes, añade una cuarta hipótesis sobre la relación entre los logros en ToM y el desarrollo del lenguaje y afirma:

Los estudios frente a esta temática han tomado dos rumbos: demostrar si el lenguaje precede a la comprensión de la falsa creencia o si es al contrario, es decir la comprensión de la falsa creencias promueve el desarrollo del lenguaje, este argumento se ha consolidado al demostrar que los adultos que han perdido la capacidad para el lenguaje son aun capaces de realizar correctamente las tareas de falsa creencias (Milligan, et alt 2007). Sin embargo también se ha contemplado la posibilidad que este relación no sea causal sino bidireccional donde las dos variables estén relacionadas con otro factor como las funciones ejecutivas. (p. 82)

Aunque parece no haber consenso en este debate, es importante resaltar que algunas investigaciones resaltan la importancia del lenguaje temprano (tanto al nivel de su aparición como de su complejidad) como un indicador predictivo del desarrollo de la ToM. De hecho, de acuerdo con Milligan et al. (2007), podemos decir que son los intercambios lingüísticos los que promueven el desarrollo de la ToM, ya que a la hora de evaluar la comprensión y superación satisfactoria de las tareas de falsa creencia, el lenguaje resulta ser un elemento indispensable ${ }^{1}$. Desde esta perspectiva, se podría predecir que las medidas de lenguaje general, o habilidades lingüísticas y de vocabulario, se relacionarían con el desempeño en tareas de falsa creencia porque proporcionan una buena indicación de la capacidad del niño para participar en la interacción social lingüística (Milligan et al., 2007).

Otro de los argumentos a favor de la estrecha relación que existe entre habilidades mentalistas y lenguaje se encuentra en estudios con poblaciones atípicas (Resches et al., 2010). Los niños con autismo, que habitualmente fallan en tareas de ToM, suelen presentar retrasos lingüísticos. Incluso analizando detenidamente las condiciones

1 Las tareas de falsa creencia han sido el instrumento tradicionalmente utilizado para evaluar la capacidad de los niños para razonar acerca de las consecuencias del comportamiento (Hale \& Tager-Flusberg, 2003). 
que hacen posible que algunos niños autistas superen las tareas básicas de ToM, la evidencia señala que el nivel de habilidad verbal constituye un indicador confiable para estimar lo que será el desempeño en dichas tareas (Tager-Flusberg \& Joseph, 2005, citado por Resches et al., 2010). Concretamente, las investigaciones realizadas en este campo han mostrado que es la sintaxis la que juega un papel importante en la adquisición de la ToM (Slade \& Ruffman, 2005).

Un estudio realizado por Paynter y Peterson (2010) demostró que la contribución de la sintaxis es mayor que la del vocabulario léxico no sólo en niños preescolares con desarrollo típico, sino también en personas con síndrome de Asperger y Autismo de Alto Funcionamiento. Estos hallazgos se apoyan en la correlación percibida entre la estructura sintáctica de las tareas de falsa creencia y el grado de comprensión y razonamiento que exhiben los niños sobre la tarea. La hipótesis sugiere que, además de la capacidad semántica puesta en práctica a la hora de comprender el significado de las palabras, en las tareas de falsa creencias estaría en juego la sintaxis, pues se requiere que el participante entienda cómo la disposición y combinación de palabras afecta el significado de las oraciones (Slade \& Ruffman, 2005).

En esta línea argumentativa, uno de los principales aspectos que Lohmann \& Tomasello (2003), encuentran en su estudio con niños con desarrollo típico, es que el lenguaje es una condición necesaria para progresar en el entendimiento de falsas creencias. Estos autores encuentran además que el entrenamiento en la sintaxis de complementos oracionales fue por sí mismo suficiente para facilitar el entendimiento de falsas creencias. De acuerdo con Resches et al. (2010), estos complementos oracionales son "construcciones complejas que consisten en una oración principal con otra insertada en ella, y que funciona como objeto gramatical del verbo en la oración principal" ( $p$. 324 - 325) (por ejemplo: María dice que el libro está en la estantería). De Villiers \& Pyers (2002), hallan además, que el dominio de las estructuras lingüísticas (directamente relacionadas con el dominio de la sintaxis) sirve de puente entre las dos líneas de desarrollo: por un lado, el desarrollo gramatical que codifica los acontecimientos y, por otro, la comprensión del comportamiento de los demás y la construcción de una teoría al respecto.

Algunas investigaciones sugieren que los niños que logran superar satisfactoriamente las tareas de falsa creencia se apoyan especialmente en el lenguaje y, en particular, en los complementos oracionales. Esta característica hablaría de un indicador principal que, al nivel del desarrollo cognitivo, anticipa en los niños la aparición del juego simbólico y, posteriormente, el desarrollo de la capacidad metarepresentacional que permite atribuir estados mentales con contenido a otros (Mebarak, Martínez \& Serna, 2009). En el estudio de Milligan et al. (2007) citado más arriba, se examinaron cinco aspectos diferentes del sistema lingüístico: habilidad lingüística general, semántica, vocabulario receptivo, sintaxis y memoria. Se encontró una relación significativa entre el rendimiento en las tareas de falsa creencia y cada uno de estos cinco tipos de habilidad lingüística, atribuyéndose $29 \%$ de varianza en la comprensión de falsa creencia a la sintaxis. Los resultados confirman la sintaxis como un predictor clave en la aparición de las habilidades mentalistas necesarias para superar tareas de falsa creencia. De ahí que, para aquellos niños que muestren retrasos en estas áreas, las intervenciones deberían dirigirse tanto a los aspectos estructurales del lenguaje (en el ámbito de la sintaxis) como a las habilidades de lenguaje figurado ${ }^{2}$. Estas formas lingüísticas figuradas hacen referencia, siguiendo a Artigas (1999), a construcciones tales como metáforas, dobles sentidos, significados implícitos y formas de cortesía, para cuyo entendimiento es necesaria una interpretación no de lo que se dice, sino de lo que se pretende o se quiere decir. Esta adquisición de los complementos oracionales con verbos de comunicación resulta ser, además, una de las pistas más confiables para explicar por qué algunos niños con autismo son capaces de superar satisfactoriamente las tareas de falsa creencia (Tager-Flusberg y Joseph, 2005; citado por Resches et al., 2010).

\section{Población de niños con síndrome de Asperger}

Desde sus descripciones iniciales se destacan, como rasgos característicos del síndrome de Asperger, un patrón de dificultades de interacción social, limitada reciprocidad socioemocional, dificultades en el uso social del lenguaje y, finalmente, presencia de intereses obsesivos y conductas repetitivas y restringidas (Ayuda-Pascual \& Martos-Pérez, 2007). No obstante, las dimensiones morfológica y sin-

2 Otros autores (Dunn \& Brophy, 2002, citado por Fisher et al., 2005), por el contrario, hacen mayor hincapié en el discurso y la conversación, argumentando que ambas son de vital importancia para entender el desarrollo de la mente de los niños, y que las propiedades de las relaciones específicas que establece el niño (por ejemplo, con los padres e iguales) predicen las diferencias individuales en el desarrollo de esta capacidad de la mente. Cabe señalar, además, que a la luz de resultados de estudios como el de Lohmann \& Tomasello (2003), tanto el intercambio de perspectivas en el discurso como el dominio de las propiedades semántico-sintácticas de los términos de estado mental, parecen realizar contribuciones independientes e igualmente importantes al desarrollo de la ToM. 
táctica del lenguaje, (no contempladas en los manuales de diagnóstico de trastornos mentales como indicadores de la posible presencia del trastorno de Asperger) conforman también algunas de las "limitaciones de naturaleza gramatical" que, indudablemente, repercuten y pueden ser indicadores de los déficits en el desempeño pragmático y comunicativo de esta población. Esto se confirma cuando notamos que las alteraciones comunicativas que presenta esta población se manifiestan especialmente en términos de déficits pragmáticos al nivel de los usos del lenguaje, más que al nivel de los aspectos estructurales.

Los estudios acerca de las relaciones que se establecen entre las tareas de falsa creencia y los aspectos estructurales del lenguaje (morfología y sintaxis), sugieren que estas tareas comúnmente utilizadas para medir habilidades de ToM son fuertemente dependientes de aspectos básicos del desarrollo del lenguaje que pueden aparecer, en ocasiones, afectados en niños con Trastorno de Espectro Autista (en adelante, TEA) (Astington \& Jenkins, 1999 citado por Whyte, Nelson \& Scherf, 2013). Es este elemento el que podría dar cuenta del fracaso que un gran porcentaje de niños con autismo presentan en la resolución de tareas de ToM. Además, aquellos niños con autismo que logran resolver tareas básicas de ToM, tienen un nivel de desarrollo del lenguaje mucho más elevado que los que no lo consiguen, aunque parecen requerir un mayor nivel lingüístico que los niños con desarrollo típico para superarlas. Por ello, autores como Fisher et al. (2005), defienden la idea de que el lenguaje puede proporcionar a los niños con TEA una ruta alternativa que les lleve a una cierta comprensión de la mente. Si este fuera el caso, la relación entre lenguaje y ToM estaría exacerbada en los niños con TEA, en comparación con los niños con desarrollo típico.

Otra característica lingüística conocida de los niños incluidos dentro de este espectro es, de acuerdo con Seung (2007), la reversión de pronombres (usar tú cuando el pronombre adecuado es yo). Rapin \& Dunn (1997), también encuentran que algunos de estos niños tienen dificultades para responder a ciertos tipos de preguntas. Entre algunas de ellas, detectan problemas para responder correctamente a preguntas por qué y cómo, así como preguntas con qué y quién, cuando los referentes no están totalmente claros en el contexto. A todo ello se le suma, como muestra el estudio de Seung (2007), que la muestra de jóvenes con Autismo de Alto Funcionamiento y síndrome de Asperger tienden a responder con mayor frecuencia a las preguntas qué por encima de las por qué, aun cuando ambas están incluidas en la misma oración. Los dos grupos utilizan, con muy poca frecuencia, marcadores intencionales durante sus discursos lo que, de acuerdo con el autor, podría ser consistente con los conocidos problemas de comprensión de intenciones que tradicionalmente se atribuyen a esta población.

\section{Relación de habilidades discursivas y narrativas con el desarrollo de la Teoría de la Mente}

Las tareas de falsa creencia utilizadas en diferentes estudios presentan una gran variedad de demandas lingüísticas en los niños; por esto es posible que el rendimiento en los distintos tipos de tareas pueda diferir en el funcionamiento lingüístico de cada niño (Milligan et al. 2007). De ahí que el input discursivo, referido a los estímulos lingüísticos a los que un niño está expuesto en un contexto comunicativo, juega un papel relevante en la emergencia de la ToM (Resches et al., 2010).

En lo que respecta a las habilidades narrativas, cabe señalar su importancia no sólo para el desarrollo de habilidades lingüísticas sino también cognitivas y sociales. La capacidad narrativa se entiende como la destreza necesaria para crear o contar una historia por medio del lenguaje, localizando diversos eventos y personales que para la persona resultan significantes (Jaramillo, 2013). Para que un niño sea capaz de elaborar una historia coherente, debe manejar las relaciones temporales y de causa-efecto, y las habilidades de ToM. De este modo, y en la línea de lo expuesto por Dodd, Ocampo \& Kennedy (2011), los estudios sobre las habilidades narrativas en niños con TEA se han enfocado en el rol de la ToM, que es interpretada como un prerrequisito para ciertos niveles de desarrollo de esta habilidad.

Kleinknecht \& Beike (2004), en este sentido, encuentran en su estudio una estrecha relación entre la capacidad de ToM de los niños y la amplitud y contenido de sus narraciones de cuentos de ficción. Debido a la importancia de las habilidades narrativas para construir la realidad y desarrollar habilidades de ToM aplicadas a la cotidianeidad, la dificultad para formular narraciones apropiadas que, en ocasiones, manifiestan los niños con Asperger, influye negativamente en su competencia socio-emocional y comunicativa (Capps, Losh \& Thurber, 2000). A continuación se presenta un estudio de caso en el que se busca examinar y comprobar algunas de las hipótesis y hallazgos identificados en torno al desarrollo morfosintáctico en población con Síndrome de Asperger. 


\section{Estudio de caso}

El estudio del desarrollo morfosintáctico de los niños con Asperger puede ayudar a clarificar la relación que se establece entre estos dominios lingüísticos y la ToM. De este modo, son varias las razones que justifican la necesidad de llevar a cabo análisis sobre las dificultades en el desarrollo del lenguaje estructural de estos niños a una edad temprana. En primer lugar, porque además de las ya conocidas alteraciones pragmáticas que presentan, la presencia de ciertos déficits en los ámbitos morfológico y sintáctico puede repercutir en su desempeño socio comunicativo. Además, y teniendo en cuenta que el caso concreto de estudio alrededor del que gira este trabajo se encuentra escolarizado en un colegio de enseñanza ordinaria, la influencia de la dimensión lingüística del niño en el plano académico es evidente (Baixauli, Roselló \& Miranda, 2004).

Numerosos estudios han tratado de investigar el desarrollo de habilidades morfosintácticas y su relación con la ToM en niños con Asperger. Sin embargo, cuando se acota la edad de estos niños a la etapa de Educación Infantil, los trabajos al respecto son más bien escasos.

En este estudio, por tanto, se propone examinar profundamente el funcionamiento lingüístico de base gramatical en un caso de un niño con Síndrome de Asperger, relacionando estos ámbitos con su desempeño en cuanto a capacidades de ToM. Se estudian además sus habilidades narrativas, que son factor clave en la ejecución de tareas que implican directamente el desarrollo de capacidades de ToM.

La idea de este trabajo no es únicamente contribuir al desarrollo de estudios de investigación sobre habilidades lingüísticas de niños Asperger, sino que además pueda utilizarse además como punto de partida para el diseño y la implementación de una posible intervención desde el contexto de escolaridad en que se encuentra inmerso.

\section{Método}

Este trabajo es un estudio de caso, desarrollado a través de una metodología de investigación observacional, para ilustrar, generalizar y representar una teoría ya existente. El carácter de la investigación es descriptivo-interpretativo, pues persigue la identificación y descripción de los diferentes factores que influyen en la cuestión objeto de estudio (Martínez, 2006).

Los criterios que orientan la selección del caso se justifican, en primer lugar, por el aliciente de conocer las características del caso, esto es, el desarrollo de los aspectos estructurales del lenguaje de este niño Asperger. Se suma la posibilidad de acceso al niño en cuestión así como al entorno en que se encuentra escolarizado. Son escasos los estudios con información extensa y cualitativa en lo que se refiere a perfiles de niños de Educación Infantil con problemas del desarrollo de aspectos formales lingüísticos.

\section{Unidad de análisis}

La unidad de análisis de este estudio es situacional, es decir, que el trabajo girará alrededor de un único sujeto con características particulares. El caso a estudiar corresponde al de un niño de 5 años y 8 meses con Trastorno de Asperger que se encuentra escolarizado en el aula de $3^{\circ}$ de Educación Infantil de un colegio público. Para su selección, el niño debía presentar una evaluación psicopedagógica formulada desde el centro educativo y un diagnóstico de síndrome de Asperger, y tanto familia como escuela debían, además, presentar las autorizaciones correspondientes.

\section{Instrumentos}

Se utilizó, en primer lugar, la Prueba de Lenguaje Oral de Navarra (PLON-R) (Aguinaga, Armentia, Fraile, Olangua y Uriz, 2004), un instrumento de screening para detectar problemas lingüísticos a edades tempranas e identificar las dimensiones que podrían estar afectadas. Esta herramienta evalúa cada uno de los ámbitos que conforman la dimensión lingüística: forma, contenido y uso, presentando además un índice de fiabilidad de 0'89. Esta información se complementó con los datos obtenidos tras la aplicación del Test Peabody (Dunn, 1987), con una fiabilidad de entre 0'77 y 0'96: lo que permitió conocer el nivel de vocabulario del niño.

La primera parte de la investigación analiza el perfil pragmático de este niño, se utilizó el sistema informático CHILDES, creado por McWhinney en 1995, para transcribir y codificar una muestra de 200 emisiones de su lenguaje espontáneo. Este instrumento proporciona el Índice de Longitud Media de Enunciado, así como la proporción de frecuencia de utilización de diferentes categorías gramaticales, obtenidos tras la transcripción de la muestra de lenguaje.

Con el fin de analizar la relación entre las habilidades de ToM y comprensión de emociones de este niño, y su desarrollo morfo-sintáctico, se utilizó el apoyo visual de una serie de historias sociales para que el niño creara un relato oral. Este análisis se justifica por las dificultades halladas en estos niños para utilizar términos mentalistas que, de acuerdo con Adams (2002), pueden producir una incapacidad para relacionar las ideas del discurso. La Tabla 1 ordena las secuencias de los relatos. Antes, se hicieron varias pruebas piloto con diferentes secuencias narrativas que permitieran seleccionar las historias sociales más adecuadas al niño. 
Tabla 1 Secuencias de los relatos

\begin{tabular}{|c|c|c|c|}
\hline & Historia social 1 & Historia social 2 & Historia social 3 \\
\hline Secuencia 1 & $\begin{array}{l}\text {-Un niño comienza a toser } \\
\text { en la mesa y se pone muy } \\
\text { enfermo. }\end{array}$ & $\begin{array}{l}\text {-Una madre pasea por la feria con su hija } \\
\text { y ven a lo lejos una tienda donde venden } \\
\text { globos. }\end{array}$ & $\begin{array}{l}\text {-Un señor entra en una habita- } \\
\text { ción donde no hay nadie. }\end{array}$ \\
\hline Secuencia 2 & $\begin{array}{l}\text {-Sus padres lo llevan al hos- } \\
\text { pital en coche. }\end{array}$ & $\begin{array}{l}\text {-La niña pide a su madre que le compre } \\
\text { un globo. }\end{array}$ & $\begin{array}{l}\text {-Deja sus gafas encima de la } \\
\text { mesa y se va. }\end{array}$ \\
\hline Secuencia 3 & $\begin{array}{l}\text {-En la habitación del hospital } \\
\text { le miran la fiebre y le dan } \\
\text { una inyección. }\end{array}$ & $\begin{array}{l}\text {-Después, a la niña se le escapa el globo y se } \\
\text { queda atrapado entre las ramas de un árbol. }\end{array}$ & $\begin{array}{l}\text {-Después viene una mujer y coge } \\
\text { las gafas. }\end{array}$ \\
\hline Secuencia 4 & $\begin{array}{l}\text {-El niño se recupera de la } \\
\text { enfermedad. }\end{array}$ & $\begin{array}{l}\text {-La niña se echa a llorar y un señor que pasa } \\
\text { por allí coge una escalera y sube al árbol a } \\
\text { recuperar el globo. }\end{array}$ & $\begin{array}{l}\text {-La mujer mete las gafas dentro } \\
\text { de un cajón y se va. }\end{array}$ \\
\hline Secuencia 5 & $\begin{array}{l}\text {-Sus padres y él vuelven a } \\
\text { casa de nuevo. }\end{array}$ & $\begin{array}{l}\text {-La niña da las gracias al señor y continúa el } \\
\text { paseo con su madre y el globo. }\end{array}$ & $\begin{array}{l}\text {-Cuando llega el señor, se da } \\
\text { cuenta de que sus gafas no están } \\
\text { encima de la mesa. }\end{array}$ \\
\hline
\end{tabular}

La construcción de la historia social tipo 1 no demanda la implicación de habilidades de ToM ni de comprensión de emociones; la historia tipo 2 sí requiere la identificación y comprensión emocional; y en la historia tipo 3 se ponen en juego las capacidades de ToM del niño. La evaluación del desempeño del niño durante la construcción de los tres tipos de historias y el análisis de las relaciones entre el desarrollo morfosintáctico y ToM, se realizó adoptando un conjunto de códigos de formato de campo (tabla 2) y teniendo siempre en cuenta la edad del niño. Se adjudicó, a cada uno de estos códigos, una valoración positiva o negativa en función del grado de desempeño del niño en cada uno de ellos a la hora de construir los tres tipos de secuencias.

Tabla 2 Códigos del formato de campo

\begin{tabular}{ll}
\hline \multicolumn{1}{c}{ Sintaxis } & \multicolumn{1}{c}{ Narrativa } \\
\hline 1. Predominio de oraciones compuestas & 7. Originalidad del argumento \\
\hline $\begin{array}{l}\text { 2. Estructura y orden de los elementos } \\
\text { 3. Coherencia de las oraciones }\end{array}$ & 8. Coherencia textual: superestructuras \\
$\begin{array}{l}\text { 4. Utilización de tiempos verbales de acuerdo con la estructura y sentido } \\
\text { de la oración }\end{array}$ & $\begin{array}{l}\text { 10. Incorporación de nexo de sucesión temporal y } \\
\text { de base causal }\end{array}$ \\
$\begin{array}{l}\text { 5. Utilización de complementos verbales de acuerdo con la estructura } \\
\text { y sentido de la oración }\end{array}$ & 11. Cohesión morfo-sintáctica \\
$\begin{array}{l}\text { 6. Utilización de pronombres de acuerdo con la estructura y sentido } \\
\text { de la oración }\end{array}$ & 12. Cohesión sintáctica \\
\hline
\end{tabular}




\section{Resultados}

Tras la aplicación de PLON-R, los resultados obtenidos informan que el niño se encuentra en un rango de "necesita mejorar" en lo que respecta a las dimensiones de "forma y contenido" (morfosintaxis y semántica), y en el intervalo de "retraso" en cuanto a la dimensión pragmática. La puntuación que el niño obtiene en el Peabody complementa la información recabada a través del PLON-R en cuanto a su desempeño en la dimensión léxica, encontrándose así en un nivel de vocabulario medio-bajo. Estas alteraciones se relacionan directamente con el Índice de Longitud Media de Enunciado obtenido con el programa CLAN (uno de los comandos que pueden utilizarse con el sistema CHILDES), que arroja un resultado de 2'74 por lo que, de acuerdo con el estudio de López, Regal \& Domínguez (2000), el niño se encontraría situado entre 24 y 29 meses. Este sistema ha permitido, también, extraer la frecuencia de uso de diferentes categorías morfológicas (figura 1). Como puede comprobarse, se detecta un uso muy limitado de algunos de estos códigos, para la edad que tiene el niño, encontrándose una escasa utilización de verbos, adjetivos, pronombres personales y preposiciones.
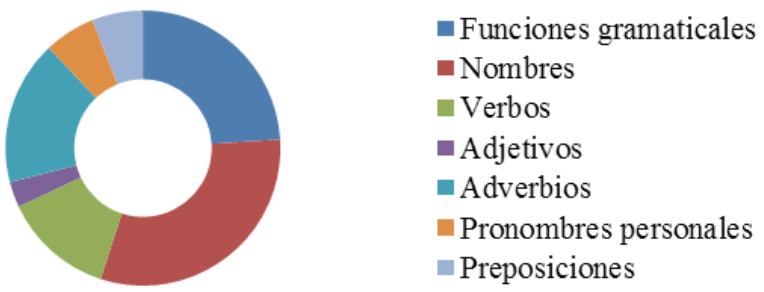

Figura 1 Proporción de utilización de categorías morfológicas

De acuerdo con los datos recabados tras la valoración de los códigos que componen el formato de campo, el mayor número de errores morfosintácticos que se aprecian en este niño con Asperger se localizan en la construcción de complementos oracionales y la utilización de pronombres personales. En el siguiente espacio se presenta la transcripción literal de las emisiones que produjo el niño durante la creación de la historia social tipo 3, secuencia en la que sí se ven implicadas las habilidades ToM:

El señor lleva gafas. Las deja encima de la mesa y se va a trabajar. Una señora viene entonces. No ve bien y coge las gafas. Luego las deja en el cajón. Entonces vuelve el señor. El señor habla, mira la mesa. Llega una mujer y abre el cajón. Entonces en el cajón están.
La utilización de secuencias narrativas como apoyo visual para evaluar estos aspectos ha permitido averiguar que, un elevado porcentaje de estos errores se produce durante la resolución de tareas que requieren la implicación directa de las habilidades de ToM (figura 2); mientras que aquella tarea del tipo 1 en la que el niño no ha necesitado poner en juego habilidades mentalistas, las dificultades de construcción de la historia han sido mucho menos notables. El número de categorías afectadas durante la elaboración de la historia tipo 2 no es tan elevado como en la historia tipo 3, pero siguen apareciendo ciertas alteraciones gramaticales y narrativas que dificultan una construcción coherente de la historia.

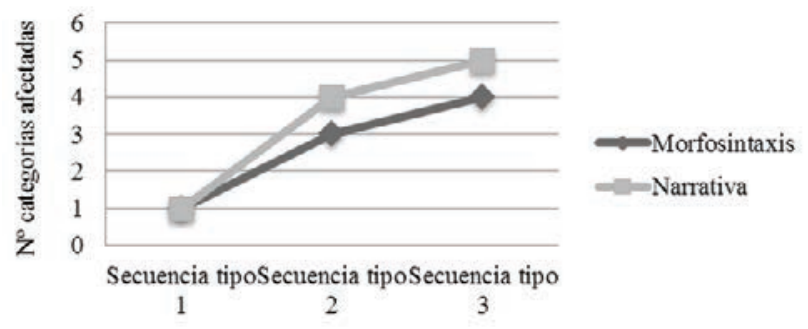

Figura 2 Categorías afectadas en cada secuencia narrativa

Consecuentemente, las narraciones más ricas y completas se han producido en aquellas historias en las que no se han visto comprometidas ni las capacidades de ToM ni las de comprensión de emociones. Dadas las manifiestas dificultades en el uso del lenguaje causal, tampoco se han apreciado en el niño inclusiones de explicaciones causales a los hechos narrados, especialmente cuando las tareas requerían un alto nivel de implicación de habilidades de ToM.

\section{Discusión}

Estos hallazgos ilustran la importancia del lenguaje para influir en la concepción de la mente de este niño con síndrome de Asperger. Sugieren, además, que el lenguaje no se limita a reflejar o comunicar nuestros pensamientos, creencias, deseos e intenciones a los demás, sino más bien que el dominio de construcciones lingüísticas específicas fomenta la capacidad para atribuir explícitamente estados mentales a uno mismo y a los demás. Esto hace del ámbito lingüístico un sistema prominente en la capacidad de los niños para desarrollar habilidades mentalistas. En esta línea, el estudio de Pascual, Aguado, Sotillo \& Masdeu (2008), también confirma que son las frases subordinadas con complementos los principales predictores de referencias reales a los deseos y creencias. 
Coincidiendo con los resultados del estudio de Mendoza \& Garzón (2012), se detectan ciertas alteraciones en este niño en lo que respecta al desarrollo sintáctico. La escasa utilización que hace el niño de diferentes categorías gramaticales conduce a la presencia de ciertas perturbaciones para consolidar un discurso rico y equilibrado morfológicamente, aspecto que se relaciona directamente con el bajo Índice de Longitud Media de Enunciado. Los datos aquí observados están en consonancia con los del estudio de Eigsti, Bennetto \& Dadlani (2007), con niños autistas, aunque, en este caso, con un grado de afectación menor. Así, el descubrimiento más relevante del trabajo que desarrollan estos autores es la producción de un lenguaje menos complejo en el grupo de niños con autismo de lo que debería esperarse para su edad.

En lo que respecta a las capacidades mentalistas, los resultados aquí encontrados están en línea con los del estudio de Kleinknecht \& Beike (2004), donde se concluye que los niños con una ToM más plenamente desarrollada se comprometen más en la narración de la historia y son capaces de transmitir realmente la esencia del relato al experimentador; ocurre el proceso contrario en aquellos niños cuyo dominio de las habilidades de ToM no es el adecuado. Por ende, para usar la narrativa con éxito, el orador tiene que considerar la perspectiva del interlocutor, poniendo en juego, por tanto, sus habilidades de ToM (Colle, Baron-Cohen, Wheelwright y Van der Lely, 2008).

La investigación sobre habilidades narrativas ha encontrado que la ToM es una condición que interviene directamente en la capacidad del niño para poder presuponer lo que el otro sabe sobre un suceso, y sobre cómo deben construirse las narraciones para que el interlocutor las comprenda bien. Así, en este trabajo se demuestra que las capacidades de atribución de estados mentales y las capacidades narrativas están fuertemente relacionadas, igual que se deduce en el estudio de Flórez, Arias \& Torrado (2011). El bajo número de inclusión de explicaciones causales encontradas ejemplariza a la perfección sus dificultades para comprender y manejar capacidades mentalistas.

La importancia de evaluar todas estas habilidades queda reflejada en el trabajo de Bennett et al. (2008), quienes defienden la necesidad de valorar los aspectos gramaticales y estructurales del lenguaje de los niños para ayudar a predecir su evolución en edades posteriores y establecer la intervención más oportuna para cada caso concreto. Lo encontrado en este estudio abre nuevas vías de exploración sugiriendo que no todos los niños con síndrome de Asperger tienen por qué adaptarse a un patrón de desarrollo de habilidades lingüísticas normalizado. Este caso es, por ende, una clara ejemplificación de la heterogeneidad lingüística que entre los niños con trastorno de Asperger es posible encontrar.

\section{Referencias}

Adams, C. (2002). Practitioner review: The assessment of language pragmatics. Journal of Child Psychology and Psychiatry, 43 (8), 973-987.

Aguinaga, G., Armentia, M., Fraile, A., Olangua, P. y Uriz, N. (2004). Prueba de Lenguaje Oral de Navarra-Revisada (PLON-R). Pamplona: Fondo de Publicaciones del Gobierno de Navarra.

Artigas, J. (1999). El lenguaje en los trastornos autistas. Revista de Neurología, 28 (2), 118-123.

Astington, J.W. \& Jenkins, J.M. (1999). A longitudinal study of the relation between language and Theory-of-Mind development. Developmental Psychology, 35 (5), 13111320.

Ayuda-Pascual, R. y Martos-Pérez, J. (2007). Influencia de la percepción social de las emociones en el lenguaje formal de niños con síndrome de Asperger o autismo de alto funcionamiento. Revista de Neurología, 44 (2), 57-59.

Baixauli, I., Roselló, B. y Miranda, A. (2004). Evaluación de las dificultades pragmáticas. Estudio de casos. Revista de Neurología, 38 (1), 69-79.

Baron-Cohen, S., Leslie, A.M. \& Frith, U. (1985). Does the autistic child have a 'theory of mind'?. Cognition, 21, 37-46.

Bennett, T. et al. (2008). Differentiating Autism and Asperger Syndrome on the basis of language delay or impairment. Journal of Autism Developmental Disorders, 38 (4), 616-625.

Bermúdez, M.E. (2009). Teorías infantiles de la mente y lenguaje: iun problema de huevo o de gallina? Revista Iberoamericana de Psicología: Ciencia y Tecnología, 2, 67-85.

Capps, L., Losh, M. \& Thurber, C. (2000). "The frog ate the bug and made his mouth sad": Narrative competence in children with Autism. Journal of Abnormal Child Psychology, 28 (2), 193-204.

Colle, L., Baron-Cohen, S., Wheelwright, S. \& Van der Lely, H.K. (2008). Narrative discourse in adults with High Functioning Autism or Asperger Syndrome. Journal of Autism and Developmental Disorders, 38, 28-40

Cutting, A.L. \& Dunn, J. (1999). Theory of Mind, emotion understanding, language and family background: Individual differences and interrelations. Child Development, 70 (4), 853-865.

De Villiers, J.G. \& Pyers, J.E. (2002). Complements to cognition: a longitudinal study of the relationship between complex syntax and false-belief-understanding. Cognitive Development, 17 (1), 1037-1060. 
De Villiers, J. (2007). The interface of language and Theory of Mind. Lingua, 117 (11), 1858-1878.

Dodd, J., Ocampo, A. \& Kennedy, K. (2011). Perspective Taking Through Narratives: Any intervention for students with ASD. Communication Disorders Quarterly, 33 (1), 23-33.

Dunn, L.M. (1987). Test de Vocabulario en Imágenes Peabody. Adaptación Española. Madrid: MEPSA. (Traducción de Santiago Pereda Marín).

Eigsti, I.M., Bennetto, L. \& Dadlani, M.B. (2007). Beyond pragmatics: morphosyntactic development in Autism. Journal of Autism Developmental Disorders, 37 (6), 10071023.

Fisher, N., Happé, F. \& Dunn, J. (2005). The relationship between vocabulary, grammar and false belief task performance in children with Autistic Spectrum Disorders and children with moderate learning difficulties. Journal of Child Psychology and Psychiatry, 46 (4), 409-419.

Flórez, R., Arias, N. y Torrado, M.C. (2011). Teoría de la Mente en tareas de falsa creencia y producción narrativa en preescolares: Investigaciones contemporáneas. Revista Colombiana de Psicología, 20 (2), 249-264.

Hale, C.M. \& Tager-Flusberg, H. (2003). The influence of language on Theory of Mind: A training study. Developmental Science, 6 (3), 346-359.

Hughes, C. \& Dunn, J. (1997). "Pretend you didn't know": Preschoolers' talk about mental states in pretend play. Cognitive Development, 12 (4), 477-499.

Jaramillo, P. (2013). El desarrollo del yo y la relación con el otro en el Trastorno del Espectro Autista. Recuperado del sitio de Internet de Universidad de Manizales: http://ridum.umanizales.edu.co:8080/xmlui/bitstream/ handle/6789/914/Jaramilo_Duarte_Paulinapdf.pdf?sequence $=1$

Kleinknecht, E. \& Beike, D.R. (2004). How knowing and doing inform an Autobiography: Relations among preschoolers' Theory of Mind, narrative, and event memory skills. Applied Cognitive Psychology, 18 (6), 745-764.

Lohmann, H. \& Tomasello, M. (2003). The role of language in the development of false belief understanding: A training study. Child Development, 74 (4), 1130-1144.

López, M., Regal, N. y Domínguez, M.T. (2000). Longitud de la oración en el desarrollo del lenguaje infantil. Revista Cubana de Pediatría, 72 (1), 27-31.

Martínez Carazo, P.C. (2006). El método de estudio de caso. Estrategia metodológica de la investigación científica. Pensamiento y gestión, 20, 165-193.

Mebarak, M., Martínez, M. y Serna, A. (2009). Revisión bibliográfico analítica acerca de las diversas teorías y programas de intervención del autismo infantil. Psicología desde el Caribe, 24, 120-146.

Mendoza, E. y Garzón, M. (2012). ¿Puede el CCC-2 diferenciar perfiles pragmáticos? Revista Chilena de Fonoaudiología, 11, 37-56.

Milligan, K., Astington, J.W. \& Dack, L.A. (2007). Language and Theory of Mind: Meta-analysis of the relation between language ability and false-belief understanding. Child Development, 78 (2), 622-646.

Pascual, B., Aguado, G., Sotillo, M. \& Masdeu, J.C. (2008). Acquisition of mental state language in Spanish children: a longitudinal study of the relationship between the production of mental verbs and linguistic development. Developmental Science, 11 (4), 454-466.

Paynter, J. \& Peterson, C. (2010). Language and ToM development in Autism versus Asperger syndrome: Contrasting influences of syntactic versus lexical/semantic maturity. Research in Autism Spectrum Disorders, 4 (3), 377-385.

Rapin, I. \& Dunn, M. (1997). Language disorders in children with autism. Seminars in Pediatric Neurology, 4, 86-92.

Resches, M., Serrat, E., Rostan, C. y Esteban, M. (2010). Lenguaje y Teoría de la Mente: una aproximación multidimensional. Infancia y Aprendizaje, 33 (3), 315-333.

Seung, H.K. (2007). Linguistic characteristics of individuals with Hign Functioning Autism and Asperger Syndrome. Clinical Linguistics and Phonetics, 21 (4), 247-259.

Slade, L. \& Ruffman, T. (2005). How language does (and does not) relate to Theory of Mind: A longitudinal study of syntax, semantics, working memory and false belief. British Journal of Developmental Psychology, 23 (1), 117141.

Tirapu-Ustárroz, J., Pérez-Sayes, G., Erekatxo-Bilbao, M. y Pelegrín-Valero, C. (2007). ¿Qué es la teoría de la mente? Revista de Neurología, 44, 479-489.

Whyte, E.M., Nelson, K. \& Scherf, K.S. (2013). Idiom, syntax and advanced Theory of Mind abilities in children with Autism Spectrum Disorders. Journal of Speech Language and Hearing Research, 57, 120-130. 